\title{
ANALYSIS OF THE INTERACTION OF SHORT-PULSE HIGH-FLUENCE RADIATION WITH TARGETS
}

\author{
R. J. Lawrence \\ Sandia National Laboratories, * Albuquerque, NM 87185
}

RECEIVED

AUG 111999

\begin{abstract}
We generally use large-scale hydrocodes to study the dynamic response of targets to hil fluence pulsed radiation loads. However, for many applications where the desired solution does not require a detailed specification of pressure- or velocity-time histories, there are simple analytic approaches that can yield surprisingly accurate results. Examples include determining either the final velocity of a radiation-driven flying plate or the impulse delivered to a structural element. These methods are all based on relatively straightforward use of conservation of mass and momentum, but they typically need one scaling-law parameter. In this context, short pulse means short compared to the characteristic time of the desired response, which allows for the phenomena to be essentially uncoupled. High fluence means that the input energy is great enough to yield vaporization or blowoff of one or more portions of the configuration. We discuss some of these methods, give examples, and suggest limitations and criteria for their use.
\end{abstract}

\section{INTRODUCTION}

Much of the impetus for the development of hydrocodes over the past several decades has come from the : requirement to investigate the vulnerability, survivability, and lethality of various targets to the effects of traditional nuclear weapons. More recently the Strategic Defense Initiative provided an additional driver for this work. A parallel effort to develop analytic methods to solve many of the same problems was also conducted, but for a number of reasons, it was less successful. The interaction phenomena that can be considered in this context include the effects of medium- and low-energy $x$ rays, visible and near-visible pulsed lasers, high-power microwaves, neutral and charged particle beams, and hypervelocity particles. The target responses that we are considering here are generally limited to dynamic mechanical effects, but do include both material and structural response phenomena. The most important issue is to determine the question that really needs answering. For example, is the target incapacitated to the point that it cannot complete its mission (lethality)? Or, is some component or subset of the overall target disabled so that mission performance is only degraded (vulnerability)? The former may be able to be answered quite simply, but the latter will likely require much more detailed analysis.

\section{APPROACH FOR ANALYTIC MODELING}

The thrust of this discussion is to clarify the conditions under which simple analytic modeling might provide adequate answers. Most of the simple models for treating dynamic mechanical response are based largely on momentum and energy conservation. They are suitable for determining integral quantities (e.g., late-time velocity or displacement) as opposed to nonintegral variables (e.g., detailed $\boldsymbol{p}(\boldsymbol{t})$ histories).

\footnotetext{
* Sandia is a multiprogram laboratory operated by Sandia Corporation, a Lockheed Martin Company, for the United States Department of Energy under Contract DE-AC04-94AL85000.
} 


\section{DISCLAIMER}

This report was prepared as an account of work sponsored by an agency of the United States Government. Neither the United States Government nor any agency thereof, nor any of their employees, make any warranty, express or implied, or assumes any legal liability or responsibility for the accuracy, completeness, or usefulness of any information, apparatus, product, or process disclosed, or represents that its use would not infringe privately owned rights. Reference herein to any specific commercial product, process, or service by trade name, trademark, manufacturer, or otherwise does not necessarily constitute or imply its endorsement, recommendation, or favoring by the United States Government or any agency thereof. The views and opinions of authors expressed herein do not necessarily state or reflect those of the United States Government or any agency thereof. 


\section{DISCLAIMER}

Portions of this document may be illegible in electronic image products. Images are produced from the best available original document. 
These approaches have the added advantage that they often lead to effective scaling laws and techniques for optimizing one or more effects. However, because of their simplicity, credibility and validation requirements mean they must be backed up with at least limited experimental data.

As an example we will look at the deposition of pulsed $x$-ray or laser radiation and determine the resultant blowoff momentum delivered to the

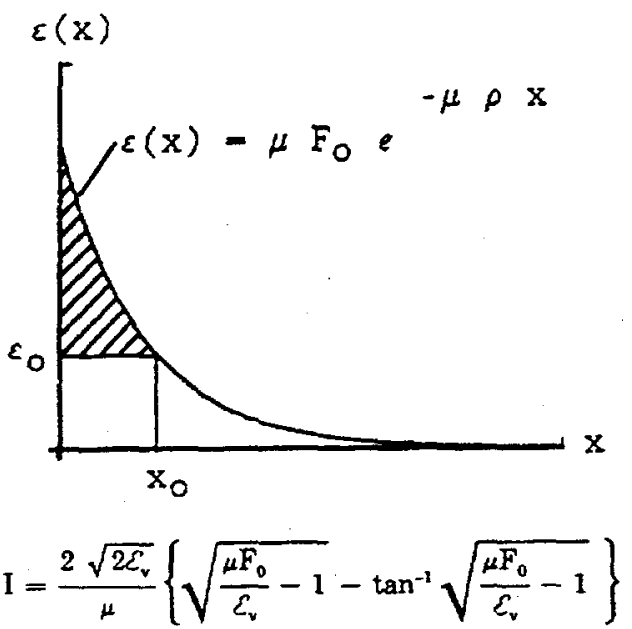

FIGURE 1. Exponential energy deposition with closed-form expression for impulse generation. $\mu$ is the material absorption coefficient: $\varepsilon_{v}$ is a specific value for the reference energy $\varepsilon_{0}$.

target. By assuming that the radiation is deposited exponentially, we can derive a particularly simple expression for target momentum or impulse, ${ }^{1}$ as illustrated in Fig. 1. Similar expressions for other classes of énergy deposition can also be developed easily.' By using non-dimensional parameters the relationship for impulse collapses onto one universal curve, as shown in Fig. 2. The latter exhibits three important features: 1) a threshold for impulse production, where the non-dimensional fluence $F_{o}^{*}$ equals one; 2) a peak impulse coupling coefficient $I^{*} / F_{o}{ }^{*}$, which is at the knee of the curve, and is indicated by the small triangles on the axes; and 3) an impulse that scales as $I \sim F_{o}{ }^{I / 2}$ at high fluences.

Early analytic models for these phenomena appeared to be less than fully successful because it was not appreciated that the regions of interest were below the knee of the curve, relatively close to the threshold. Here the curve is very steep, and small uncertainties in fluence lead to large differences in impulse. Because the overall shape of the curve was not well understood at that time, these differences were perceived as model errors.

The only unknown parameters in this simple model are the material absorption coefficient $\mu$ and the reference energies $\varepsilon_{v}$ or $\varepsilon_{v}$, which are generally

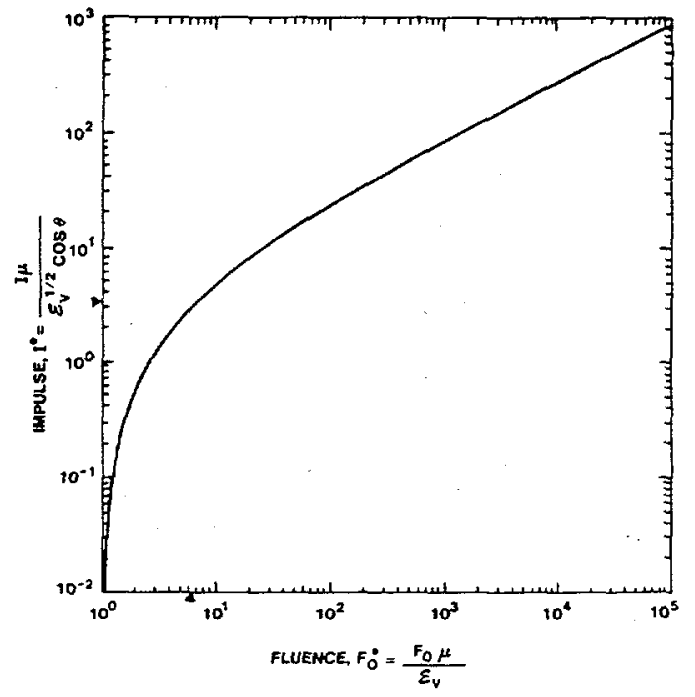

FIGURE 2. Non-dimensional relation between fluence and impulse. $\theta$ is the angle of incidence.

defined as material decomposition energies. Depending on the circumstances, effective values for these parameters can usually be chosen. Hence this and related models have been termed effective properties models. ${ }^{2}$ Although there do not seem to be any simple "rules of thumb" for determining the reference energy $\varepsilon_{0}$, the absorption coefficients often scale with the radiation photon energy $h v$ and the atomic number $Z$ as $\mu \sim(h v)^{-3}$ and $\mu \sim Z^{4 \text { or } 5}$, as long as the jumps at the photoelectric absorption edges are neglected.

\section{MODEL VALIDATION}

As suggested above, such simple models require experimental confirmation for validation and to establish credibility. One related application that turned out particularly well was the study of laserdriven tamped flyer plates. ${ }^{3}$ The relevant configuration involved flyers several $10 \mathrm{~s}$ of microns thick that were plated on the ends of long optical fibers. A high-power laser pulse was sent down the fiber, and the inner surface of the flyer was vaporized, but fully tamped by the fiber. The 
resultant flyer velocity was measured and compared to predictions of a model derived in a manner similar to that described above. Figure 3 shows this comparison for aluminum flyers. As can be seen, the agreement is excellent from near the threshold to well into the high-fluence limit, as well as for several different flyer diameters.

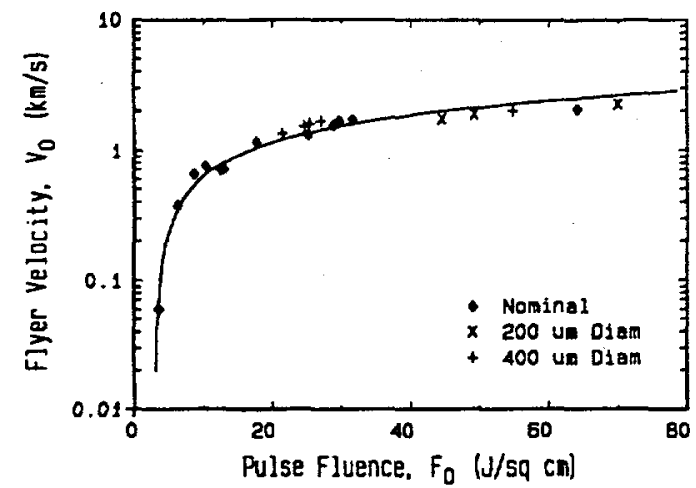

FIGURE 3. Comparison of model predictions with experimental measurements for laser-driven tamped aluminum flyers. The flyers were $25 \mu \mathrm{m}$ thick, and the laser pulse width was $25 \mathrm{~ns}$. The fact that flyer diameter has no appreciable effect on flyer velocity indicates that the response is one-dimensional.

\section{APPLICATION: X-RAY-DRIVEN IMPULSE}

One of the major applications for these types of models is the determination of impulsively driven buckling thresholds for various types of structures. This approach has been widely used as a measure of target lethality to pulsed $x$-ray loads.

We can make this problem particularly simple by using curves such as Fig. 2, along with material absorption coefficients $\boldsymbol{\mu}$ and decomposition energies $\boldsymbol{\varepsilon}_{\boldsymbol{r}}$, to plot iso-impulse curves for materials of interest. As shown in Figs. 4 and 5, we usually plot radiation fluence as a function of photon energy. We have used two materials, a generic heat shield material, quartz phenolic, and a middle atomic number metal, iron. The latter may not be representative of a typical structural material, but it does illustrate the use of the simple model to guide the development of system requirements and optimization. In these curves, the discontinuities arise from the photoelectric absorption edges, and the dashed line is a mapping of the impulse generation threshold onto this plane. Table 1 shows several impulse levels that are representative of the requirements to induce structural buckling in different types of structures. These lethality levels, depending on the structure, vary by almost two orders of magnitude. To determine a pulsed $x$-ray lethality requirement, we simply read possible fluence and photon energy pairs from the appropriate iso-impulse curve.

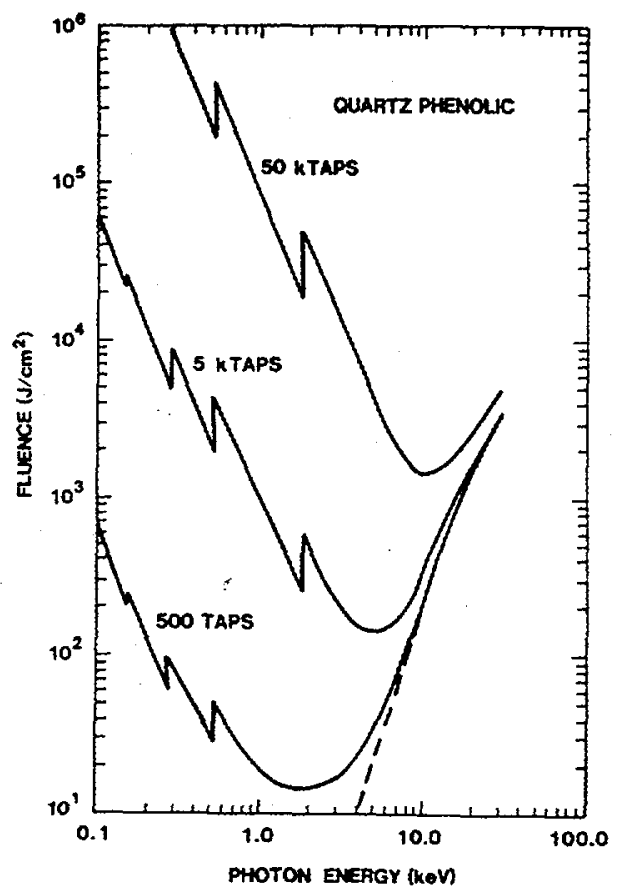

FIGURE 4. Iso-impulse curves for a typical heat shield material, quartz phenolic.

Table 2 provides several examples of this process. Suppose we wish to deliver a relatively low impulse of 500 taps to a quartz phenolic target. From Fig. 4, we see that $20 \mathrm{~J} / \mathrm{cm}^{2}$ at a photon energy of $1 \mathrm{keV}$ will accomplish the task. If the material were a mid- $Z$ metal such as iron, Fig. 5 shows that the required fluence would be three times greater, at $60 \mathrm{~J} / \mathrm{cm}^{2}$. For a more interesting example, 5 ktaps could provide a lethal buckling load to a light structure such as a satellite. At a photon energy of $1 \mathrm{keV}, 1000$ and $5000 \mathrm{~J} / \mathrm{cm}^{2}$ would be required for the same two materials. However, if we could increase the photon energy to $4 \mathrm{keV}$, the same objective could be achieved with roughly an order of magnitude lower fluence-140 and $300 \mathrm{~J} / \mathrm{cm}^{2}$ respectively. These simple examples illustrate the use of analytic models to 
establish or determine system-level parameters for accomplishing specific tasks. They also show how,

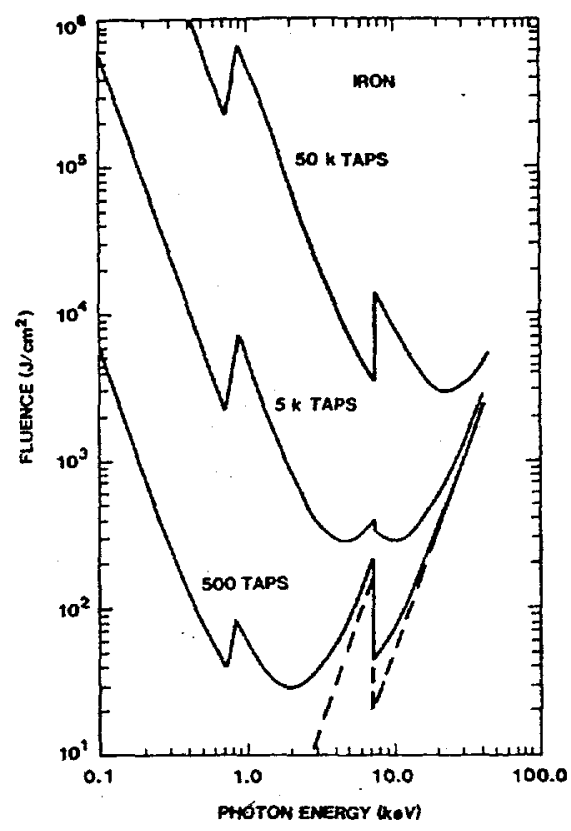

FIGURE 5. Iso-impulse curves for a typical mid-Z metal, iron (steel).

Table 1. Typical Lethality Response Levels

\begin{tabular}{ll}
\hline $\begin{array}{l}\text { Light-weight structures } \\
\text { (e.g., satellites) }\end{array}$ & 1 to $10 \mathrm{ktaps}{ }^{\text {a }}$ \\
$\begin{array}{c}\text { Medium-weight structures } \\
\text { (e.g., airframes) }\end{array}$ & 10 to $30 \mathrm{ktaps}$ \\
$\begin{array}{c}\text { Robust structures } \\
\text { (e.g., reentry vehicles) }\end{array}$ & 30 to $80 \mathrm{ktaps}$ \\
\hline
\end{tabular}

${ }^{a} 1$ tap $=1$ dyne-s $/ \mathrm{cm}^{2}$

Table 2. Example Calculations

\begin{tabular}{ccc}
\hline \multicolumn{2}{c}{$\begin{array}{c}\text { Required Load } \\
\text { and Photon Energy }\end{array}$} & Required Fluence \\
\hline 500 taps @ $1 \mathrm{keV}$ & $\mathrm{QP}^{\mathrm{b}}$ & $20 \mathrm{~J} / \mathrm{cm}^{2}$ \\
& $\mathrm{Fe}^{\mathrm{c}}$ & $60 \mathrm{~J} / \mathrm{cm}^{2}$ \\
$5 \mathrm{ktaps} @ 1 \mathrm{keV}$ & $\mathrm{QP}$ & $1000 \mathrm{~J} / \mathrm{cm}^{2}$ \\
& $\mathrm{Fe}$ & $5000 \mathrm{~J} / \mathrm{cm}^{2}$ \\
$5 \mathrm{ktaps} @ 4 \mathrm{keV}$ & $\mathrm{QP}$ & $140 \mathrm{~J} / \mathrm{cm}^{2}$ \\
& $\mathrm{Fe}$ & $300 \mathrm{~J} / \mathrm{cm}^{2}$ \\
\hline
\end{tabular}

${ }^{\mathrm{b}} \mathrm{QP}=$ Quartz Phenolic ${ }^{\mathrm{c}} \mathrm{Fe}=$ Iron (steel) in principle, modest changes in system design can lead to dramatic reductions in performance requirements, at least for pulsed $\mathrm{x}$-ray-driven interactions. Other similar applications, such as the modeling of pulsed-laser-driven flyers, will have different characteristics, but these analytic models will all lead to more complete pictures of the relevant parametric space. Although the explicit answers generated by these models are often only approximate, they clearly indicate response trends in a way that cannot be achieved easily with point calculations using more detailed and expensive numerical analyses.

\section{CONCLUSIONS}

We have described some relatively simple analytic models that can be used successfully for certain problems that are often studied with more elaborate methods such as hydrocodes. In particular, for studies related to the determination of lethality requirements, these simple approaches are often more than adequate. This is especially true if we are looking for integrated late-time velocities or displacements in contrast to detailed pressure or velocity histories. In fact, many applications (e.g., laser-driven flyers) can use these analytic models without major limitations.

However, for detailed analyses related to system vulnerability or survivability, complex numerical hydrocodes are still needed. They are the only tools that can provide time-resolved output for all desired quantities, and they are able to examine more detailed sensitivities as well as higher order phenomena. On the other hand, they are often difficult and time consuming to employ.

\section{REFERENCES}

1. Lawrence, R. J., "The Equivalence of Simple Models for Radiation-Induced Impulse," in Shock Compression of Condensed Matter-1991, edited by S. C. Schmidt, et al., Elsevier Science Publishers, Amsterdam, pp. 785-788 (1992).

2. Lawrence, R. J., An Effective Properties Model for Pulsed Radiation Interactions (SAND88-0245), Sandia National Laboratories, Albuquerque, NM (1988).

3. Lawrence, R. J. and W. M. Trott, Int. J. Impact Engng., 14, 439-449 (1993). 